\title{
Exploration of the Lysis Mechanisms of Leukaemic Blasts by Chimaeric T-Cells
}

\author{
David Laurin, ${ }^{1,2,3}$ Virna Marin, ${ }^{4}$ Ettore Biagi, ${ }^{4}$ Irene Pizzitola, ${ }^{4}$ Valentina Agostoni, ${ }^{4}$ \\ Géraldine Gallot, ${ }^{5,6}$ Henri Vié, ${ }^{5}$ Marie Christine Jacob, ${ }^{2,3,7}$ Laurence Chaperot, ${ }^{4}$ \\ Caroline Aspord, ${ }^{1,2,3}$ and Joël Plumas ${ }^{5}$ \\ ${ }^{1}$ Etablissement Français du Sang, 29 av du Maquis du Grésivaudan, BP35, 38701 La Tronche, France \\ ${ }^{2}$ Inserm, U823, Immunobiologie et Immunotherapie des cancers, 38706 La Tronche, France \\ ${ }^{3}$ Université Joseph Fourier, 38041 Grenoble, France \\ ${ }^{4}$ Department of Paediatrics, Centro di Ricerca Matilde Tettamanti, University of Milano-Bicocca, San Gerardo Hospital, \\ 20052 Monza, Milan, Italy \\ ${ }^{5}$ CHU Nantes, Hôtel Dieu, UTCG, 44093 Nantes, France \\ ${ }^{6}$ Inserm, U892, 44093 Nantes, France \\ ${ }^{7}$ Laboratoire d'Immunologie CHU Grenoble, 38043 Grenoble, France
}

Correspondence should be addressed to David Laurin, david.laurin@efs.sante.fr

Received 3 December 2009; Accepted 18 March 2010

Academic Editor: Hanchun Yang

Copyright () 2010 David Laurin et al. This is an open access article distributed under the Creative Commons Attribution License, which permits unrestricted use, distribution, and reproduction in any medium, provided the original work is properly cited.

\begin{abstract}
Adoptive transfer of specific cytotoxic T lymphocytes (CTL) and Cytokine Induced Killer Cells (CIK) following genetic engineering of T-cell receptor zeta hold promising perspective in immunotherapy. In the present work we focused on the mechanisms of anti-tumor action of effectors transduced with an anti-CD19 chimaeric receptor in the context of B-lineage acute lymphoblastic leukemia (B-ALL). Primary B-ALL blasts were efficiently killed by both z-CD19 CTL and z-CD19 CIK effectors. The use of death receptor mediated apoptosis of target cells was excluded since agonists molecules of Fas and TRAIL-receptors failed to induce cell death. Perforin/granzyme pathway was found to be the mechanism of chimaeric effectors mediated killing. Indeed, cytolytic effector molecules perforin as well as granzymes were highly expressed by CTL and CIK. CD19 specific stimulation of transduced effectors was associated with degranulation as attested by CD107 membrane expression and high IFN- $\gamma$ and TNF- $\alpha$ release. Moreover inhibitors of the perforin-based cytotoxic pathway, $\mathrm{Ca}^{2+}$-chelating agent EGTA and Concanamycin A, almost completely abrogated B-ALL blast killing. In conclusion we show that the cytolysis response of z-CD19 chimaeric effectors is predominantly mediated via perforin/granzyme pathway and is independent of death receptors signaling in primary B-ALL.
\end{abstract}

\section{Introduction}

Allogeneic hematopoietic stem cell transplantation constitutes the main curative treatment of hematological malignancies and disorders. The potency of the graft versusleukemia effect varies widely depending on the type of leukemia. For B-lineage acute lymphoblastic leukemia (BALL), the graft versus-leukemia effect is modest and consequently disease relapse after transplantation is a major contributor to treatment failure [1].

Adoptive cellular therapy such as donor lymphocyte infusions achieves poor remission rates in patients with BALL and is associated with a high incidence and severity of graft-versus-host disease morbidity and mortality [2]. Adoptive immunotherapy targeting chosen antigens selectively expressed by leukaemic targets should separate graft-versusleukemia and graft-versus-host disease.

Ex vivo genetic engineering of $\mathrm{T}$ cells and Cytokineinduced killer cells (CIK) using gene-transfer technology should overcome these barriers and allow the production of large numbers of leukaemia-specific effector cells. Chimaeric receptors are generated by joining the heavy and light chain variable regions of a monoclonal antibody, expressed as a single-chain Fv molecule, to the cytoplasmic T-cell receptor zeta [3]. 
Chimaeric immunoreceptors that use antibody-derived single-chain variable domains bypass the requirement for antigen processing and presentation by HLA molecules. Modified effectors exhibit specific lysis and cytokine secretion upon exposure to tumor cells expressing the respective target antigen [4] and shown protection in murine tumor models [5].

CD19-specific chimaeric Cytotoxic T lymphocytes (CTL) [6-8], z-CD19 natural killer cells (NK) [9], or z-CD19 CIK [10] effectors specifically recognize and kill CD19+ leukemia/lymphoma cells and primary B-ALL blasts. Nevertheless, the mechanisms of this lysis has never been studied.

Two main pathways of CTL-mediated cytotoxicity, that is, granule exocytosis mediated by perforin/granzymes and the death receptor-mediated Fas/Fas ligand (FasL) system, have been identified [11]. More recently, tumor necrosis factor-related apoptosis-inducing ligand (TRAIL) was found to induce apoptosis in a variety of tumor cells and leukemia [12]. Mechanisms that trigger apoptosis in target cells are shared by CTL and NK cells.

Death receptors are members of the tumor-necrosis factor (TNF) receptor superfamily and include a subfamily that is characterized by an intracellular domain - the death domain [13]. Decoy receptors are closely related to the death receptors but lack a functional death domain. Death receptors are activated by their natural ligands from the TNF family. Ligands bind to their respective death receptorssuch as CD95, TRAIL-R1 (TNF-related apoptosis-inducing ligand-R1 or Death Receptor 4), and TRAIL-R2 (DR5), then the death domains attract the intracellular adaptor protein FADD (Fas-associated death domain protein, also known as $\mathrm{MORT}_{1}$ ), which, in turn, recruits the inactive proforms of certain members of the caspase protease family. In contrast to the DR4 and DR5 receptors, two other cell-surface TRAILreceptors, DcR1 (Decoy Receptor 1 or TRAIL-R3) and DcR2 (TRAIL-R4), lack a functional death domain and cannot transduce a proapoptotic signal. These "decoy receptors" compete with DR4 and DR5 for TRAIL binding.

Apoptosis of target cells is also induced by the granule exocytosis pathway. Upon binding of an effector to a target cell (by CTL-receptor and antigen on the target cell), the content of the granules is released. These cytolytic granules contain a number of proteins: perforin, a pore forming protein, and granzymes A and B, serine proteases, which are the main effector molecules of the granule-mediated cell death.

In the present work we show that tumor killing by CTL and CIK cells transduced with zeta-CD19 receptor (zCD19) is predominantly mediated via the perforin/granzyme pathway and is independent of death receptors signaling in primary B-ALL.

\section{Materials and Methods}

2.1. Cell Lines and Primary Lymphoma Cells. Cell lines were obtained from the American Type Culture Collection (LGC Standards, Molsheim, France). We used SupB15 (ALL-B) and Raji (Burkitt's lymphoma) $\mathrm{CD} 19^{+}$leukemia cell lines and the CD19- Jurkat (ALL-T). They were maintained in RPMI-1640 Glutamax (Invitrogen, Cergy Pontoise, France) supplemented with $10 \%$ fetal calf serum, supplemented with sodium pyruvate, gentamycin, and nonessential amino acids.

Bone marrow cells and peripheral blood mononuclear cells (PBMC) were collected from patients with B-ALL at diagnosis who signed informed consent forms. Flow cytometry analysis showed that, in all tested cases, blast infiltration was $>80 \%$ and $100 \%$ of the blasts which expressed the CD19 antigen. All leukemia samples were cryopreserved and subsequently thawed to be used in the experiments.

2.2. Plasmids and Retrovirus Production. The variable domains of monoclonal antibody anti-CD19 fmc63 were cloned as single-chain $\mathrm{Fv}(\mathrm{scFv})$ molecules in frame with $\mathrm{CH} 2-\mathrm{CH} 3-\mathrm{CD} 28 \mathrm{tm}$-zeta in the SFG-retroviral construct by Martin Pule, UCL, London, UK. The retroviral supernatant was produced by FuGENE 6 (Roche Diagnostic S.p.A., Italy)-mediated cotransfection of 293-T cells with the MoMLV gag-pol expression plasmid pEQ-PAM3(-E) (kindly provided by Martin Pule), the RD114 env expression plasmid pRDF (kindly provided by Dr Yasu Takeuchi) and the SFGanti-CD19 CR vector. Supernatants containing retrovirus were harvested 48 hours and 72 hours after transfection, immediately frozen in dry ice, and stored at $-80^{\circ} \mathrm{C}$ until use. $293 \mathrm{~T}$ cells were used to titrate virus concentration.

2.3. Generation of Effectors and Retroviral Transduction. EBVSpecific CTLs and CIKs Cells were prepared from PBMC obtained after centrifugation of fresh blood on a density gradient using Ficoll-Hypaque (Eurobio, Les Ulis, France).

EBV-specific CTLs were produced as previously reported [14] PBMCs $\left(2 \times 10^{6}\right)$ were cocultured with $5 \times 10^{4}$ gamma-irradiated (40 Gy) autologous EBV lymphoblastoid cell lines (LCLs) per well in a 24-well plate. Starting on day 10 , the responder cells were restimulated weekly with irradiated LCLs at a responder-to-stimulator ratio of $4: 1$. From day 14, $40 \mathrm{IU} / \mathrm{mL}$ recombinant human interleukin2 (IL-2) (Proleukine, Chiron) was added twice a week. Twenty-four hours to three days after the third stimulation, the CTLs were transferred to a 24-well nontissue culture plates (BD) precoated with $3.7 \mu \mathrm{g} / \mathrm{cm}^{2}$ of the recombinant fibronectin fragment FN CH-296 (RetroNectin; TaKaRa BioEurope, Gennevilliers, France). Transduction was performed in culture medium supplemented with $40 \mathrm{UI} / \mathrm{mL}$ IL2 and incubation for 48 hours at $37^{\circ} \mathrm{C}$ and $5 \% \mathrm{CO}_{2}$. Then, the viral supernatant was replaced by fresh medium and a fourth stimulation was performed on day 24 .

CIK cells were prepared as described previously [10] Briefly, PBMC were resuspended in medium completed with $1000 \mathrm{U} / \mathrm{mL}$ recombinant human IFN-g (Sigma). The next day, IL-2 and anti-CD3 (Orthoclone OKT3, Janssen-Cilag) were added at $500 \mathrm{IU} / \mathrm{mL}$ and at $50 \mathrm{ng} / \mathrm{mL}$, respectively. Cells were cultured at the concentration of $3 \times 10^{6}$ cells $/ \mathrm{mL}$. Fresh medium and $300 \mathrm{IU} / \mathrm{mL}$ IL-2 were added weekly during culture. The transduction process was analogous to the one used for CTLs, except for IL-2, that was used at $600 \mathrm{IU} / \mathrm{mL}$. 
2.4. Flow Cytometric Analysis. The following affinitypurified mouse monoclonal fluorescent conjugated antibodies were used: anti-CD3, anti-CD45, antigranzyme A, antigranzyme B and isotypic controls (BD Biosciences, le Pont de Claix, France), anti-CD95 and anti-CD19 (Beckman Coulter), and antiperforine (Diaclone). Unconjugated antiDR4 (HS101), DR5 (HS201), DcR1 (HS301), and DcR2 (HS402) were from Alexis (Lausen, Switzerland). Their indirect staining was performed with a goat antimouse-PE conjugated polyclonal $\mathrm{F}\left(\mathrm{ab}^{\prime}\right)_{2}$ antibody (Beckman Coulter).

Granzymes and perforin intracellular staining was perform following permeabilization using $10 \mathrm{~min}$. incubation with BD-Fixation/Permeabilization solution from BDCytofix/Cytoperm kit and incubation in PermWash buffer.

Detection of the genetically modified zeta-transfectant was performed by the goat antihuman $(\mathrm{GAH}) \operatorname{IgG}(\mathrm{H}+\mathrm{L})$ $\mathrm{F}\left(\mathrm{ab}^{\prime}\right)_{2}$ fragment polyclonal antibodies (Beckman Coulter).

Down expression of CD45 was used to gate leukemia blasts from nonleukaemic cells. B cell origin of leukaemic gated cells was confirmed by CD19 expression.

All labeling was performed in the dark at room temperature. Analyses were performed using a FACSCanto II flow cytometer and Diva software (BD Biosciences).

2.5. Assay for Determining Sensitivity to Death ReceptorInduced Apoptosis and Cytotoxicity. Target cells were labeled with $50 \mu \mathrm{Ci}$ chromium 51 for 1 hours at $37^{\circ} \mathrm{C}$ and $5 \% \mathrm{CO}_{2}$. After 3 washes, labeled targets were plated in 96 wells plates at $10^{5}$ cells/well for apoptosis assay and $5 \times 10^{4}$ cells/well for cytotoxic assay.

The sensitivity of cells to Fas- and TRAIL-mediated apoptosis was investigated using $500 \mathrm{ng} / \mathrm{mL}$ agonistic antiCD95 (IgM clone CH11; Alexis Biochemicals, San Diego, CA) and $500 \mathrm{ng} / \mathrm{mL}$ recombinant human soluble TRAIL (KillerTRAIL; Alexis Biochemicals). Isotype IgM antibody was used as control (500 ng/mL, Beckman Coulter). Apoptosis was assayed following 4 and 18 hours of incubation at $37^{\circ} \mathrm{C}$ and $5 \% \mathrm{CO}_{2}$.

For cytotoxicity, effector cells were added at various effector to target ratios. Final volumes were $200 \mu \mathrm{L}$ and were incubated for 4 hours at $37^{\circ} \mathrm{C}$ and $5 \% \mathrm{CO}_{2}$.

Subsequently, $35 \mu \mathrm{L}$ supernatant was removed from each well and counted by the Top Count NXT (Perkin Elmer) scintillation counter to determine experimental chromium 51 release. Spontaneous release was obtained from wells receiving target cells and medium only, and total release was obtained from wells receiving $1 \mathrm{mg} / \mathrm{mL}$ Saponine (Sigma).

Percentage-specific apoptosis was calculated by the following formula: $\%$ apoptosis $=100 \times($ experimental release - spontaneous release)/(total release - spontaneous release).

Percentage-specific cytotoxicity was calculated by the following formula: $\%$ cytotoxicity $=100 \times$ (experimental release - spontaneous release)/(total release - spontaneous release).

2.6. Determination of Degranulation and Intracellular Cytokine Staining. Chimaeric effectors cells were stimulated with SupB15 cell line (1:3 ratio) for 5h30. Anti-CD107a and CD107b FITC antibodies (BD Biosciences) were added in the medium at the beginning of the stimulation in the presence of $0.7 \mu \mathrm{L} / \mathrm{mL}$ GolgiStop (BD Biosciences) and $1 \mu \mathrm{L} / \mathrm{mL}$ GolgiPlug (BD Biosciences). Cells were then labeled with anti-CD3 and goat antihuman (GAH) IgG $(\mathrm{H}+\mathrm{L})$ $\mathrm{F}\left(\mathrm{ab}^{\prime}\right)_{2}$ antibodies. Then permeabilization of the cells was performed using Cytofix/Cytoperm kit (BD Biosciences). IFN- $\gamma$ and TNF- $\alpha$ intracellular staining was then performed (BD Biosciences). Degranulation and cytokine production was analyzed on $\mathrm{CD}^{+} \mathrm{GAH}^{-}$or $\mathrm{CD}^{+} \mathrm{GAH}^{+} \mathrm{T}$ cells.

\section{Results}

3.1. Primary B ALL Blast Are Sensitive to z-CD19 CTL and CIK-Mediated Cytolysis. CTL anti-EBV and CIK cells transduced with zeta-CD19 receptor were controlled and were similar to our previous publications (see supplementary data). The zeta-CD19 receptor transduced effectors were used to assay primary B-ALL blasts lysis. A strong cytotoxic activity was observed against all blasts assayed (Figure 1).

Thus, primary B-ALL blasts are sensitive to the killing by anti-CD19-z CTL and anti-CD19-z CIK effectors.

\subsection{Poor Expression of Death Receptors and Ligand Sensibility} by B ALL Blasts. Since death receptor expression is a prerequisite for susceptibility of target cells to receive the apoptosis signal by death ligands expressed by effector cells, we investigated Blast cell surface expression by flow cytometry.

Expression of Fas was observed on 100\% of Jurkat and Raji cell lines and $60 \%$ of SupB15 (Figure 2(a)). In contrast, all primary B-ALL blasts studied were almost negative for Fas expression, with a frequency range of $1.5 \%$ to $7.5 \%$ (Figure 2(b)).

TRAIL death inducing receptors DR4 and DR5 were expressed by Jurkat and Raji with more than $49 \%$ and $65 \%$ $\mathrm{DR}^{+}$cells, respectively and $100 \% \mathrm{DR}^{+}$for both cell lines. More than $60 \%$ of SupB15 expressed DR5 but no expression of DR4 was observed. Primary B-ALL blasts expressed heterogeneously DR4 and DR5. DR4 was expressed by $1.9 \%$ to $14.5 \%$ of cells. DR5 was more strongly expressed by leukaemic cells with a range of $3.3 \%$ to $40.8 \%$ of blasts expressing this molecule. TRAIL decoy receptors DcR1 (TRAIL-R3) and DcR2 (TRAIL-R4) were not expressed by Jurkat and SupB15, whereas $40 \%$ of Raji expressed DcR1. Very interestingly, B-ALL blasts expressed DcR1 and DcR2 proportionally to death receptors, the leukemia expressed the most DR4, and DR5 expressed the highest levels of decoy receptors DcR1 and DcR2.

The absence of Fas expressed by blasts should result in the resistance of blast cells to apoptosis triggered by FasL expressed by effectors. The proportional coexpression of TRAIL-death receptors with decoy receptors could lead to the absence of TRAIL-mediated apoptosis susceptibility by B-ALL cells.

In order to confirm the resistance of B-ALL blast to cytolysis induced by death receptors, we used the agonist antibody anti-Fas clone $\mathrm{CH} 11$ and soluble recombinant human TRAIL 


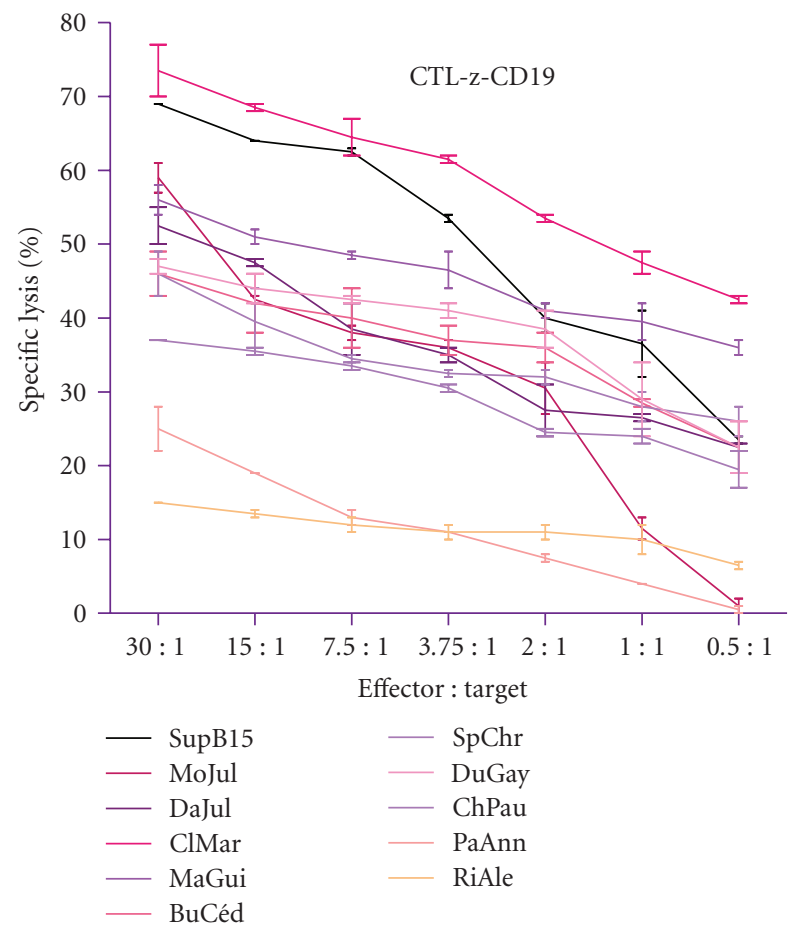

(a)

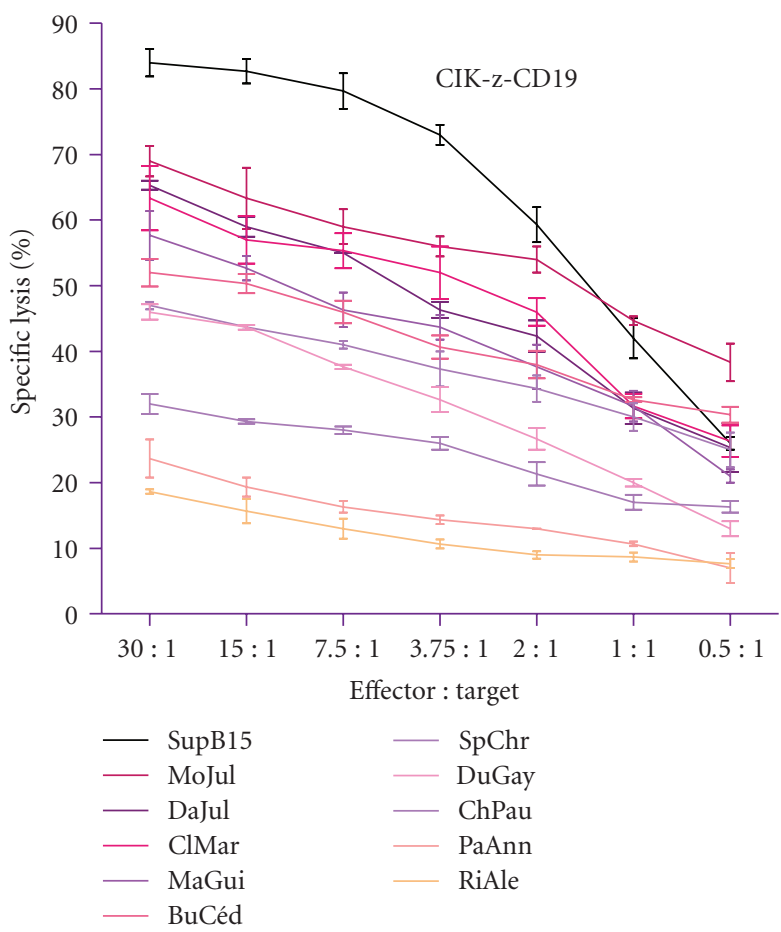

(b)

FIgURE 1: Killing of SupB15 (positive control) and tumor cells. The experiment is representative of 5 independent experiments. Similar results were produced with 3 donors of CTL and 2 donors of CIK.

(killerTRAIL) molecule on these cells (Figure 3). Apoptosis of Jurkat positive control was induced after treatment by anti-Fas and TRAIL-receptors apoptosis agonists. SupB15 was weakly sensitive to TRAIL, but not to Fas. Raji was weakly sensitive to Fas but not to TRAIL. As anticipated from phenotyping study, blasts were not sensitive to apoptosis induced by Fas and very weakly or not to TRAIL-Receptors Death induction.

We concluded that primary B-ALL blasts were not sensitive to death receptor-induced killing or very weakly. These results showed that effector cells cannot use Fas/FasL and TRAIL/TRAIL-R pathways to kill primary B-ALL leukaemic targets.

\section{3. $z$-CD19 CTL and CIK-Express Mediators of Cytotoxicity} and Express Membrane CD107 Granule Exocytosis-Associated Molecule upon B Cell Stimulation. To go further into the elucidation of effector mechanisms, we then analyzed the expression of perforin, granzyme-A, and granzyme-B by the effectors. We also compared the expression of these cytotoxicity-associated molecules from the transduced positive populations versus not transduced CTL and CIK. As shown in Figure 4(a), CTL and CIK expressed high amounts of granzymes and perforin. Retroviral transduced cells showed no alteration in the expression of perforin and granzyme-A and -B (Figure 4(a)).

We concluded that chimaeric effectors express molecules implicated in the perforin/granzyme pathway and that the transduction do not affect the expression of these molecules.
We next directly examined the degranulation of antiCD19-z chimaeric effectors by the CD107 degranulation assay. This functional assay was coupled to the cytokine release assay for IFN- $\gamma$ and TNF- $\alpha$. In order to analyze only the specific response linked to the CD19 molecule expressed by target cells, effectors were stimulated by EBV negative B cell line SupB 15 but also by primary B-ALL blasts. Nontransduced CTL and CIK were not stimulated by target cells so that the response analyzed is only the transfectantspecific stimulation.

The coculture of anti-EBV-z-CD19-CTL and z-CD19CIK effectors with $\mathrm{CD}_{1}{ }^{+}$targets was associated to a high increase in CD107 surface expression as compared to nontransduced effectors (Figure 4(b)), showing granules exocytosis. CD19-specific stimulation of transduced effectors induced high secretion of both IFN- $\gamma$ and TNF- $\alpha$ cytokines. As expected nontransduced anti-EBV and CIK revealed only a small background of cytokine production.

Altogether, results from perforin and granzymes expression with the CD107 degranulation assay highly support the hypothesis of perforin/granzyme pathway used by effectors. Moreover, intracellular cytokine staining confirmed transduced CTL and CIK functionality associated with B-ALL blasts target recognition and killing by effectors.

3.4. Inhibition of Perforin/Granzyme Pathway Block $z$ CD19 Chimaeric Effectors Cytolysis. In order to confirm the involvement of perforin/granzyme pathway, we used specific inhibitors. The exocytosis of lytic granules is 


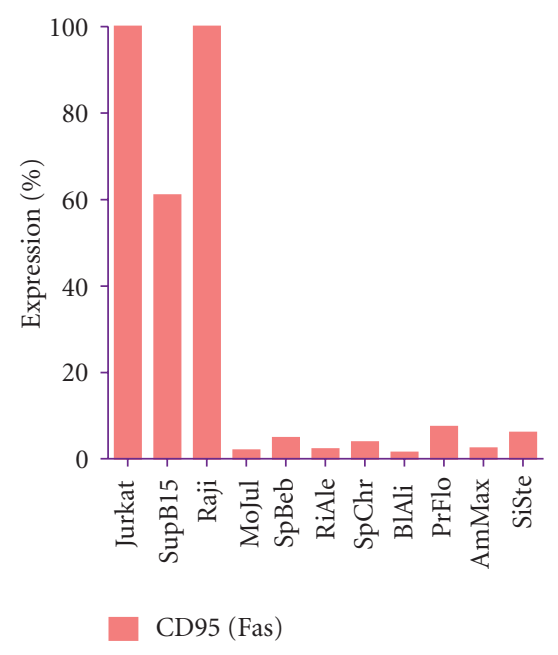

(a)

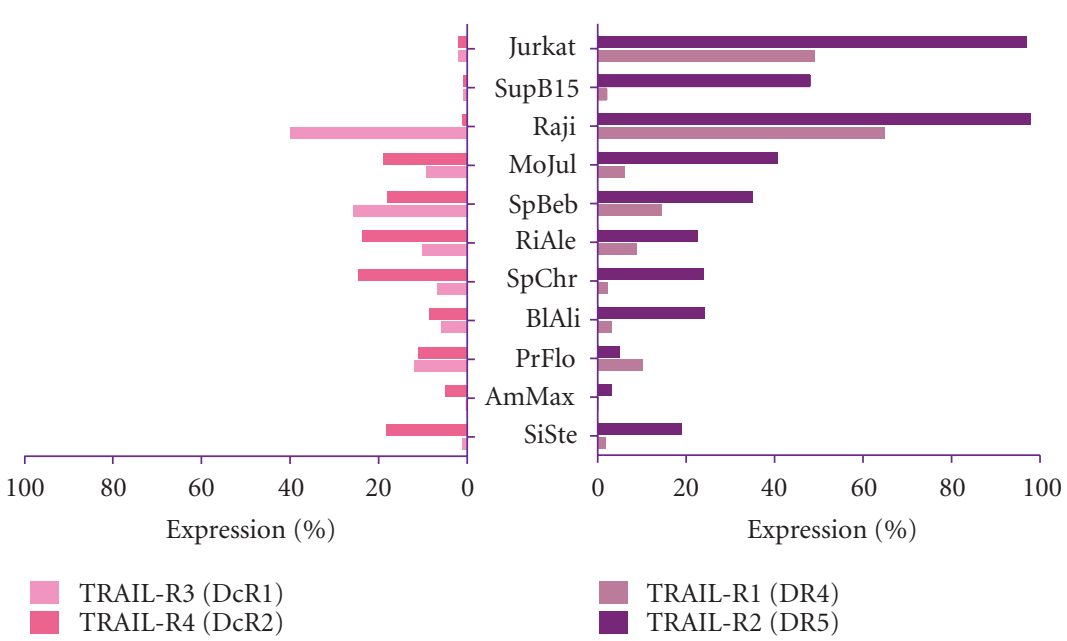

(b)

Figure 2: Death receptors expressed by Jurkat, SupB15, or Raji cell lines and B-ALL blasts. (a) Percentages of cells expressing Fas. (b) Right histograms represent percentage of cells expressing TRAIL-R1 or -R2 agonists death receptors (DR4 or DR5) and left histogram decoy antagonist TRAIL-R3 or -R4 receptors (DcR1 or DcR2).

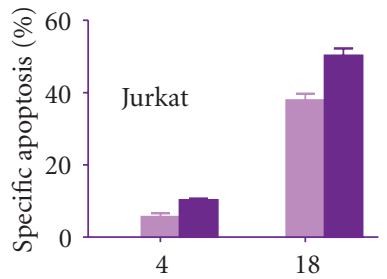

(h)

(a)

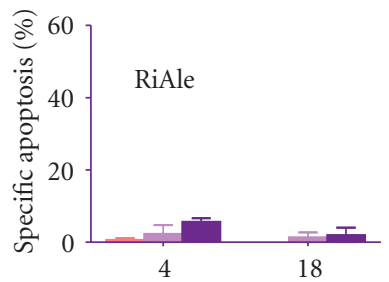

(h)

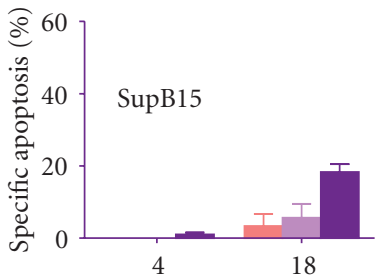

(h)

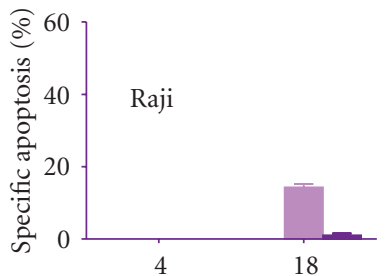

(h)

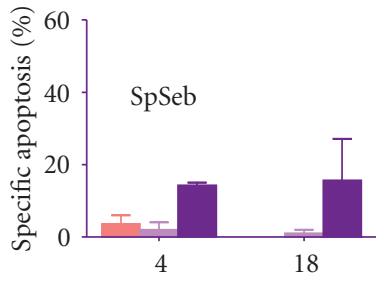

(h)

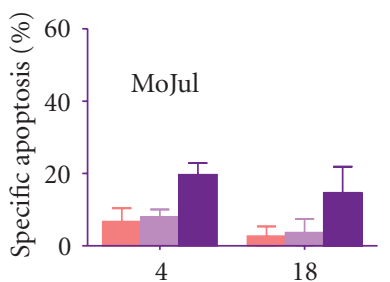

(h)

$$
\begin{aligned}
& \text { - } \lg \\
& \text { - Anti-Fas (CH11) } \\
& \text { - KillerTRAIL }
\end{aligned}
$$

(f) (c)

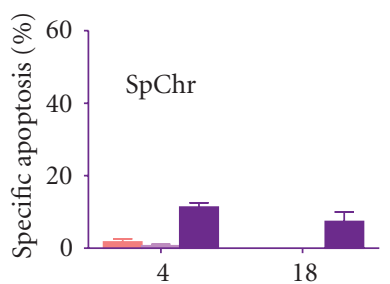

(h)

$$
\begin{aligned}
& \text { lg } \\
& \text { - Anti-Fas (CH11) } \\
& \text { - KillerTRAIL }
\end{aligned}
$$

(g) (d)

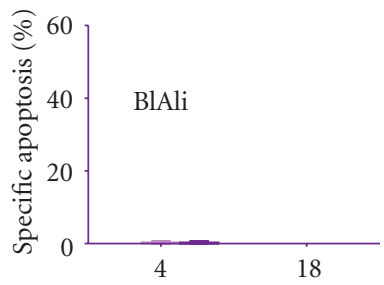

(h)

a $\lg$

Anti-Fas (CH11)

- KillerTRAIL

(h)

FIGURE 3: Apoptosis induced on Jurkat, SupB15, or Raji cell lines and B-ALL blasts by agonist antibody anti-Fas (clone CH11) or recombinant human TRAIL (killerTRAIL) measured after 4 hours and 18 hours exposition. Isotype control (Ig) is indicated.

$\mathrm{Ca}^{2+}$-dependent. We used EGTA chelating agent of divalent cations including $\mathrm{Ca}^{2+}$ to block granzymes exocytosis in the presence of $\mathrm{Mg}^{2+}$ (used to allow adherence between effectors and targets). Inhibition of perforin/granzyme pathway were also performed by Concanamycin A who degrades perforin by increasing the $\mathrm{pH}$ of lytic granules and inhibits perforinbased cytotoxic activity.

Anti-EBV-z-CD19-CTL and z-CD19-CIK efficiently killed SupB15 and Raji (Figure 5) but were inhibited by the presence of EGTA/ $\mathrm{MgCl}_{2}$ or Concanamycin A, confirming the use of perforin/granzyme pathway by effectors to kill their targets. More interestingly, primary B-ALL blasts were efficiently killed by transduced CTL and CIK effectors but the killing was abrogated in the presence of inhibitors of degranulation or of perforin.

In conclusion, we have demonstrated that chimaeric CTL and CIK effectors killed B-ALL primary blasts via the perforin/granzyme pathway. 

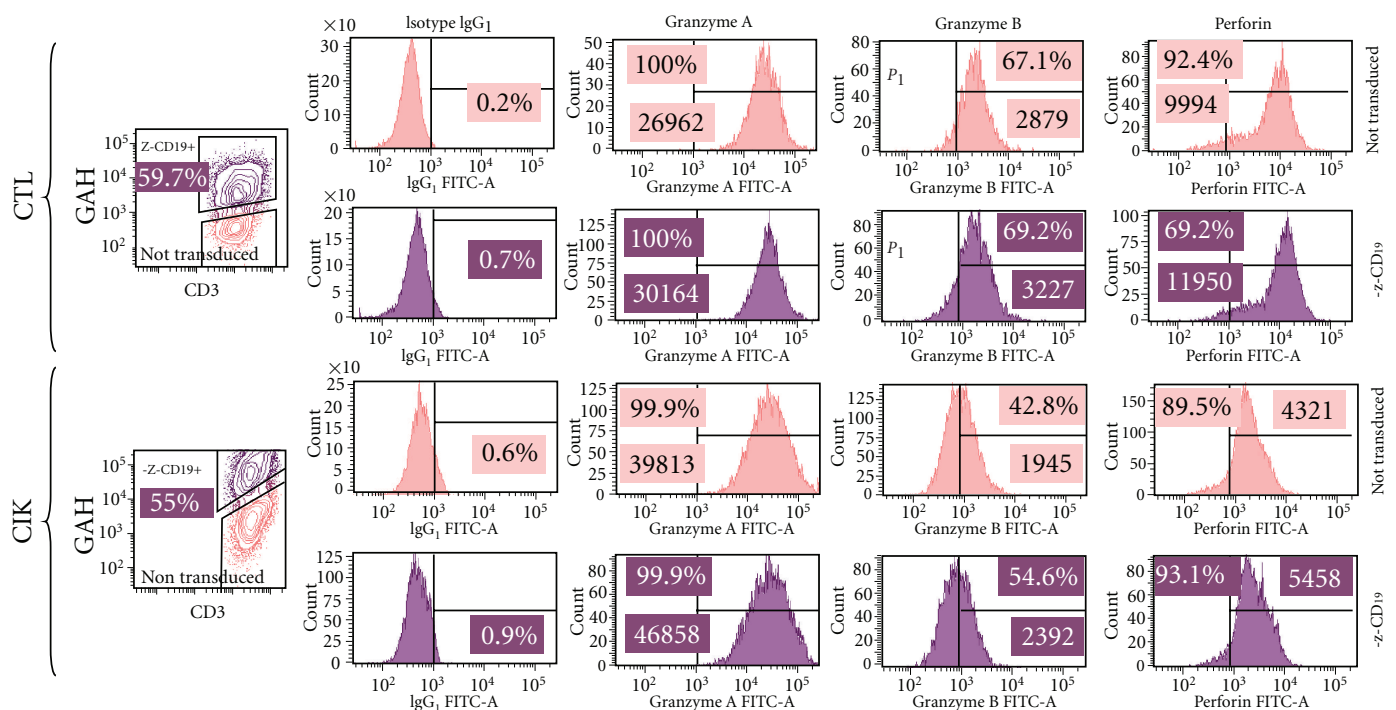

(a)
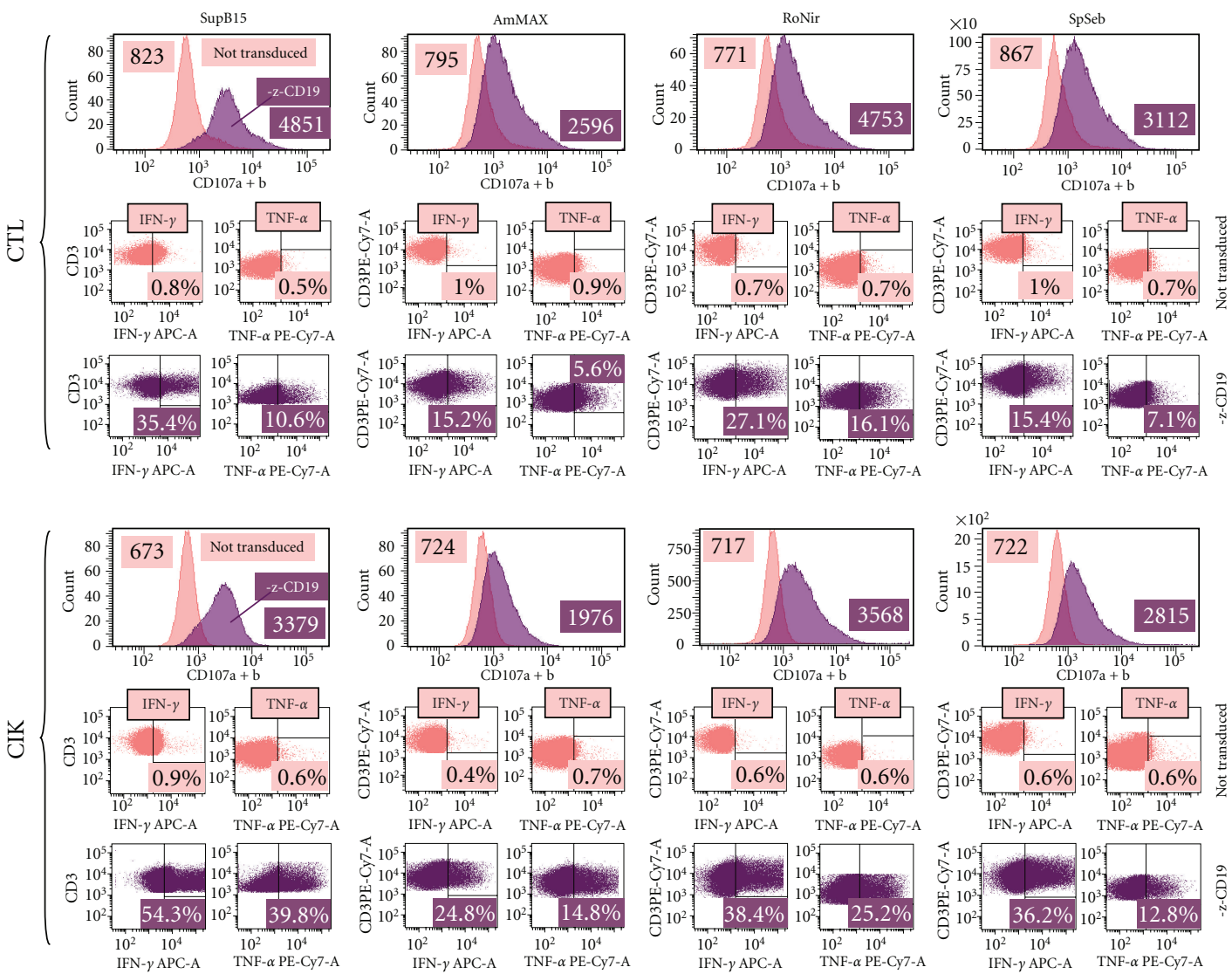

(b)

FIgURE 4: z-CD19 chimaeric effectors express mediators of cytotoxicity. (a) CTL and CIK effectors were stained with antigranzyme A, -granzyme B or -perforin antibodies. Transfectant expressions were detected by a goat antihuman IgG (GAH) labeling on $\mathrm{CD}^{+}$cells. Percentages and MFI are indicated from $\mathrm{CD}^{+}$cells. (b) CTL and CIK effectors were cocultured in the presence of SupB15 CD19+ stimulating cells or Primary B-ALL blasts (AmMax, RoNir and SpSeb). Degranulation of effectors was analyzed by anti-CD107a and -CD107b antibodies gated on $\mathrm{CD}^{+}$cells. Similarly, IFN- $\gamma$ and TNF- $\alpha$ specifically produced by responding effectors were analyzed on $\mathrm{CD}^{+}$gated cells. Similar results were produced with 2 donors of CTL and CIK. 


\section{Discussion}

Our data demonstrate that redirecting the specificity of anti-EBV CTL and CIK via a CD19-specific chimaeric immunoreceptor efficiently kills CD19+ leukaemic and lymphoma lines, as well as primary B-ALL cells. Targeting CD19 with genetically modified effectors is a strategy that has shown its potent killing efficacy on primary B-ALL. The effectors used were $\mathrm{T}$ cells activated by the $\mathrm{CD} 3$-specific antibody OKT3 [8], anti-virus specific CTL $[6,7]$, Natural Killer cells [9], or CIK [10]. In the present study we used EBV-specific CTL and CIK. Both effectors efficiently lyse tumors but the mechanisms by which chimaeric effector cells mediate killing of B-ALL blasts have not been studied until now. Tumor cell sensibility and effector-mediated lysis mechanisms are important to evaluate in order to improve the immunomonitoring of $\mathrm{T}$ cells and NK cells responses following immunotherapy. The lysis of tumor targets is mediated by cell death induced by distinct pathways $[13,14]$. Tumor escape may in part be associated to defective signaling allowing evasion to effectors functionality. The mechanisms of effector-mediated lysis are therefore also important to elucidate tumor escape strategies.

The Fas-FasL pathway is a classic effector mechanism that CTL and NK cells use to lyse target cells [15]. More recently, TRAIL was found to induce apoptosis in a variety of tumor cells and leukemia [12] upon binding with its death-inducing receptors, DR4 and DR5. At the cell surface, TRAIL binding reflects a competition between death receptors (DR4 and DR5) and so-called 'decoy receptors' (DcR1 and DcR2), that are receptor homologs but cannot signal to FADD. So we first analyzed death receptors expressed by B-ALL blasts and the apoptosis susceptibility of primary leukemia following exposition to Fas and TRAIL-receptor agonists. Our data indicate that primary B-ALL blasts poorly express Fas and are not susceptible to Fas agonist-induced apoptosis. The resistance of leukaemic cells towards Fas-mediated apoptosis found in this study is in line with previous data describing leukemia cell resistance to Fas ligation [16, 17]. Resistance to Fas ligation can be associated to different mechanisms in leukemia such as over expression of proto oncogenes or secretion of soluble Fas. In the present work, it seems that lack of Fas expression on the membrane of B-ALL blasts could explain the resistance to Fas pathway. Cell surface expression analysis of TRAIL receptors showed that primary B-ALL cells express the death-inducing receptors DR4 and/or DR5 but also the decoy receptors DcR1 and/or DcR2, potentially leading to a competition associated with an absence of death signaling. In line with this hypothesis, the use of human recombinant TRAIL on B-ALL cells failed to induce blasts apoptosis. Data from primary precursor Bcell ALL showing that TRAIL was only modestly effective in this tumor type was previously reported [18]. In contrast to our findings, authors reported that the poor TRAIL activity reflected a poor expression of death receptors as well as decoy receptors. This could be explained by the use of western blot analysis instead of more sensitive flow cytometry used in the present study and availability of more specific (monoclonal) antibodies for TRAIL-receptors expression analysis. Taken together, expression analysis of death-inducing receptors and agonists susceptibility demonstrates that primary B-ALL blasts are almost not sensitive to Fas and TRAIL-mediated apoptosis. As a consequence, specific chimaeric effectors lysis of B-ALL blasts cannot use the FasL/Fas or TRAIL/TRAILreceptors pathways.

In order to investigate the perforin/granzyme pathway, we first analyzed the expression of the cytolytic effector molecules. Anti-EBV CTL and CIK effector cells highly expressed perforin as well as granzyme A and B. Comparison of z-CD19 transduced effectors to nontransduced cells demonstrates that receptor modifications of both antiEBV CTL and CIK are not associated to an alteration in the expression of cytolytic effector molecules. High expression of cytolytic effector molecules argues in favor of perforin/granzyme pathway all the more since some experiments showed that granzymes and perforin expression correlates with cytolytic activity [19]. We then analyzed transduced effectors functionality via the chimaeric immunoreceptor z-CD19 following stimulation by SupB15 cell line but also by primary B-ALL blasts. Degranulation is a necessary prerequisite to perforin-granzyme-mediated killing. Upon effectors activation, preformed cytotoxic granules move toward the outer membrane of the cell in a polarized fashion toward the site of antigen presentation. After reaching the plasma membrane, the membrane of the granule and the plasma membrane merge, releasing the contents of the granule into the extracellular space. At this time, the CD107a and CD107b proteins from the granule membrane are accessible at the cell surface for specific antibody binding [20]. Our data indicate that both genetically modified effectors anti-EBV CTL and CIK highly increased CD107 surface expression following CD19 specific stimulation. The upregulation of surface CD107 attests the degranulation of z-CD19 chimaeric effectors. This functional assay was completed by the analysis of IFN- $\gamma$ and TNF- $\alpha$ release. The production of these proinflammatory cytokines is associated with the effectors function and killing. CD19specific stimulation of anti-EBV CTL and CIK transduced effectors induced high secretion of both IFN- $\gamma$ and TNF$\alpha$ cytokines showing high functionality of genetic modified responding cells.

Then we show that cytotoxicity mediated by CD19specific human chimaeric anti-EBV CTL as well as CIK are $\mathrm{Ca}^{2+}$-dependent, since addition of the $\mathrm{Ca}^{2+}$-chelating agent EGTA to the assay medium resulted in complete abrogation of cytotoxicity.

The exocytosis of lytic granules is $\mathrm{Ca}^{2+}$ dependent but the up-regulation of FasL on the cell surface of effectors requires also extracellular $\mathrm{Ca}^{2+}[21]$. Since in the present work we have demonstrated that primary B-ALL are not susceptible to the death induced by Fas pathway, we could conclude that the cytotoxicity mediated by the z-CD19 effectors is perforin/granzyme-dependent. Moreover, the cytotoxicity mediated by z-CD19 anti-EBV CTL and CIK was almost completely inhibited by treatment with a potent inhibitor of the perforin-based cytotoxic pathway, Concanamycin A.

In conclusion the findings reported in the present work show that the cytolysis response of z-CD19 chimaeric 


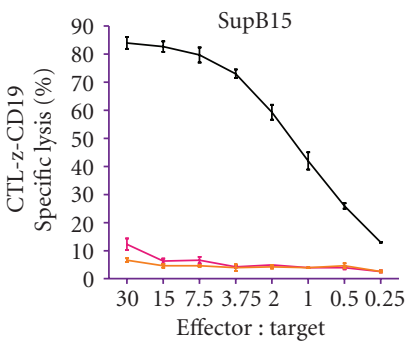

(a)

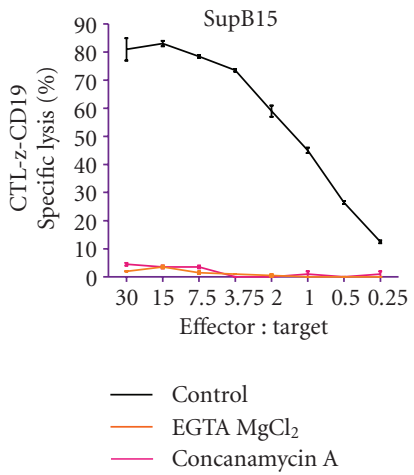

(e)

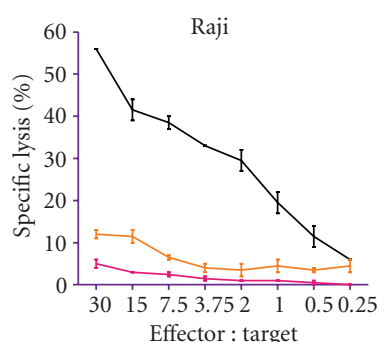

(b)

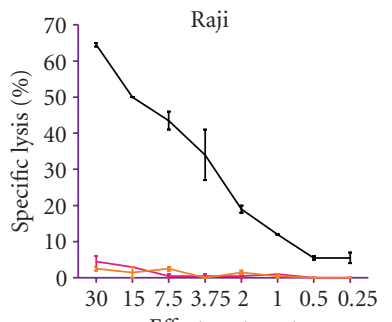

Effector : target

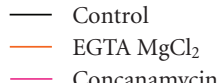

- Concanamycin A

(f)

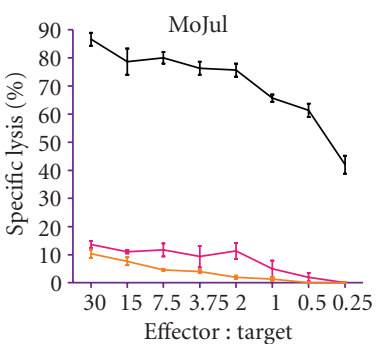

(c)

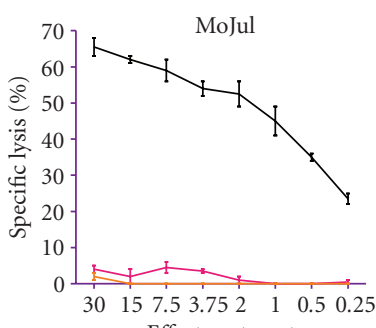

Effector : target

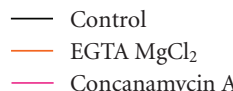

(g)

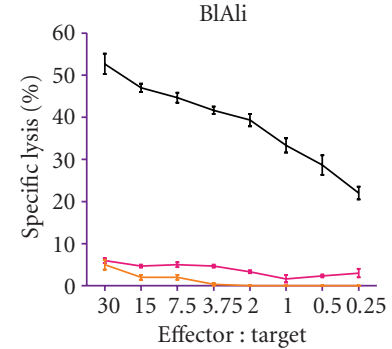

(d)

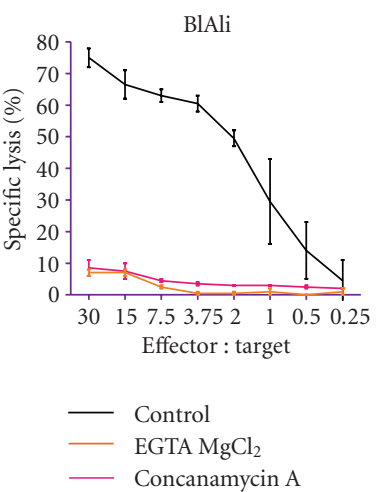

(h)

FIgURE 5: Specific Lysis of z-CD19 chimaeric effectors is inhibited in the presence of perforin/granzyme inhibitors. Cytolytic activity of effectors was analyzed against SupB15 or Raji cell lines and B-ALL blasts (control) or in the presence of EGTA/ $\mathrm{MgCl}_{2}$ or Concanamycin A.

effectors is predominantly mediated via perforin/granzyme pathway and is independent of death receptors signaling in primary B-ALL. This has important implications since in mouse models of allogeneic bone-marrow transplantation, it was shown that donor $\mathrm{T}$ cells mediate graft versus host disease primarily through the FasL effector pathway, whereas the graft-versus-leukaemia activity was mediated through the perforin pathway [22]. The present work has important implications on the monitoring of $\mathrm{z}$-CD19 engineered effector cells therapy against $\mathrm{B}$ leukemia.

\section{Abbreviations}

CTL: Cytotoxic T lymphocytes

CIK: Cytokine Induced Killer Cells

B-ALL: B-lineage acute lymphoblastic leukemia.

\section{Acknowledgments}

Funding for this work was provided by the European Union Sixth Framework Programme and AIRC 2007 (4069) Ministero della Salute-Direzione Generale della Ricerca Scientifica e Tecnologica. The use of chimeric T-cell receptors (ChTCRs) for the therapy of haematological high-risk diseases" and "Progetto Integrato Oncologia 2006", Ministero della Salute-Direzione Generale della Ricerca Scientifica e Tecnologica.

\section{References}

[1] C. H. Pui, M. Schrappe, R. C. Ribeiro, and C. M. Niemeyer, "Childhood and adolescent lymphoid and myeloid leukemia," Hematology, pp. 118-145, 2004.

[2] A. W. Loren and D. L. Porter, "Donor leukocyte infusions for the treatment of relapsed acute leukemia after allogeneic stem cell transplantation," Bone Marrow Transplantation, vol. 41, no. 5, pp. 483-493, 2008.

[3] E. Biagi, V. Marin, G. M. P. G. Attianese, E. Dander, G. D'Amico, and A. Biondi, "Chimeric T-cell receptors: new challenges for targeted immunotherapy in hematologic malignancies," Haematologica, vol. 92, no. 3, pp. 381-388, 2007.

[4] M. A. Pule, K. C. Straathof, G. Dotti, H. E. Heslop, C. M. Rooney, and M. K. Brenner, "A chimeric T cell antigen receptor that augments cytokine release and supports clonal expansion of primary human T cells," Molecular Therapy, vol. 12, no. 5, pp. 933-941, 2005.

[5] R. J. Brentjens, E. Santos, Y. Nikhamin, et al., "Genetically targeted $\mathrm{T}$ cells eradicate systemic acute lymphoblastic leukemia xenografts," Clinical Cancer Research, vol. 13, no. 18, pp. 54265435, 2007.

[6] C. Roessig, S. P. Scherer, A. Baer, et al., "Targeting CD19 with genetically modified EBV-specific human T lymphocytes," Annals of Hematology, vol. 81, supplement 2, pp. S42-S43, 2002.

[7] L. J. N. Cooper, Z. Al-Kadhimi, L. M. Serrano, et al., "Enhanced antilymphoma efficacy of CD19-redirected influenza MP1-specific CTLs by cotransfer of T cells modified to present influenza MP1," Blood, vol. 105, no. 4, pp. 16221631, 2005. 
[8] L. J. N. Cooper, M. S. Topp, L. M. Serrano, et al., "T-cell clones can be rendered specific for CD19: toward the selective augmentation of the graft-versus-B-lineage leukemia effect," Blood, vol. 101, no. 4, pp. 1637-1644, 2003.

[9] C. Imai, S. Iwamoto, and D. Campana, "Genetic modification of primary natural killer cells overcomes inhibitory signals and induces specific killing of leukemic cells," Blood, vol. 106, no. 1, pp. 376-383, 2005.

[10] V. Marin, E. Dander, E. Biagi, et al., "Characterization of in vitro migratory properties of anti-CD19 chimeric receptor-redirected CIK cells for their potential use in B-ALL immunotherapy," Experimental Hematology, vol. 34, no. 9, pp. 1219-1229, 2006.

[11] M. Barry and R. C. Bleackley, "Cytotoxic T lymphocytes: all roads lead to death," Nature Reviews Immunology, vol. 2, no. 6, pp. 401-409, 2002.

[12] S. H. Kaufmann and D. P. Steensma, "On the TRAIL of a new therapy for leukemia," Leukemia, vol. 19, no. 12, pp. 21952202, 2005.

[13] F. H. Igney and P. H. Krammer, "Death and anti-death: tumour resistance to apoptosis," Nature Reviews Cancer, vol. 2, no. 4, pp. 277-288, 2002.

[14] D. Chowdhury and J. Lieberman, "Death by a thousand cuts: granzyme pathways of programmed cell death," Annual Review of Immunology, vol. 26, pp. 389-420, 2008.

[15] D. Kagi, B. Ledermann, K. Burki, et al., "Cytotoxicity mediated by $\mathrm{T}$ cells and natural killer cells is greatly impaired in perforin-deficient mice," Nature, vol. 369, no. 6475, pp. 31-37, 1994.

[16] H. G. Otten, W. G. J. van Ginkel, A. Hagenbeek, and E. J. Petersen, "Prevalence and clinical significance of resistance to perforin- and FAS-mediated cell death in leukemia," Leukemia, vol. 18, no. 8, pp. 1401-1405, 2004.

[17] K. Uno, T. Inukai, N. Kayagaki, et al., “TNF-related apoptosisinducing ligand (TRAIL) frequently induces apoptosis in Philadelphia chromosome-positive leukemia cells," Blood, vol. 101, no. 9, pp. 3658-3667, 2003.

[18] K. Clodi, D. Wimmer, Y. Li, et al., "Expression of tumour necrosis factor (TNF)-related apoptosis-inducing ligand (TRAIL) receptors and sensitivity to TRAIL-induced apoptosis in primary B-cell acute lymphoblastic leukaemia cells," British Journal of Haematology, vol. 111, no. 2, pp. 580-586, 2000.

[19] A. Hombach, H. Kohler, G. Rappl, and H. Abken, "Human $\mathrm{CD} 4+\mathrm{T}$ cells lyse target cells via granzyme/perforin upon circumvention of MHC class II restriction by an antibody-like immunoreceptor," Journal of Immunology, vol. 177, no. 8, pp. 5668-5675, 2006.

[20] M. R. Betts, J. M. Brenchley, D. A. Price, et al., "Sensitive and viable identification of antigen-specific CD $8+T$ cells by a flow cytometric assay for degranulation," Journal of Immunological Methods, vol. 281, no. 1-2, pp. 65-78, 2003.

[21] B. Lowin, C. Mattman, M. Hahne, and J. Tschopp, "Comparison of Fas(Apo-1/CD95)- and perforin-mediated cytotoxicity in primary T lymphocytes," International Immunology, vol. 8, no. 1, pp. 57-63, 1996.

[22] C. Schmaltz, O. Alpdogan, K. J. Horndasch, et al., "Differential use of Fas ligand and perforin cytotoxic pathways by donor T cells in graft-versus-host disease and graft-versus-leukemia effect," Blood, vol. 97, no. 9, pp. 2886-2895, 2001. 


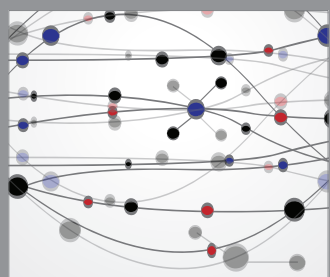

The Scientific World Journal
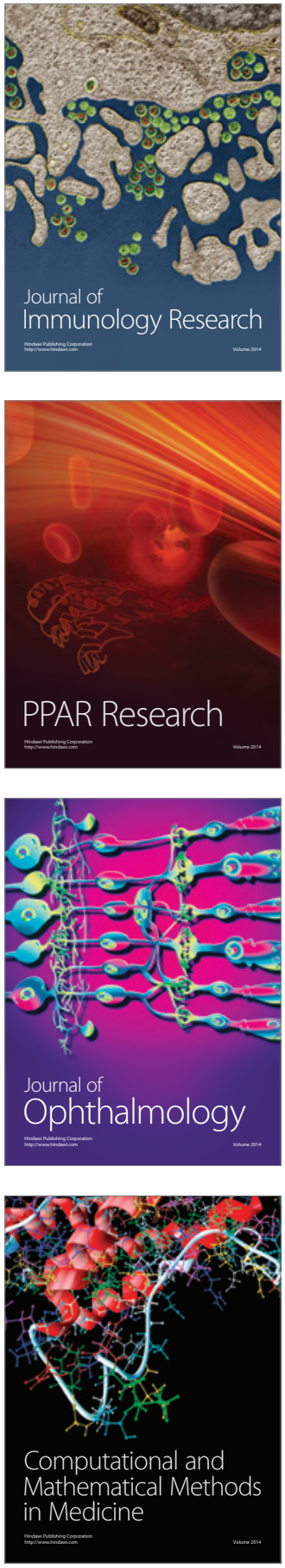

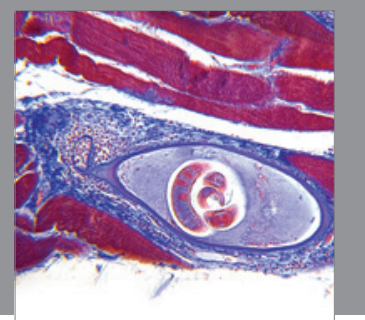

Gastroenterology

Research and Practice
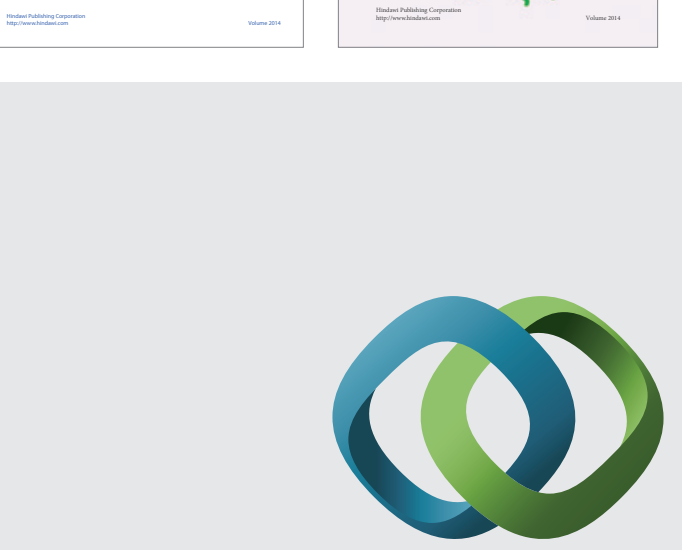

\section{Hindawi}

Submit your manuscripts at

http://www.hindawi.com
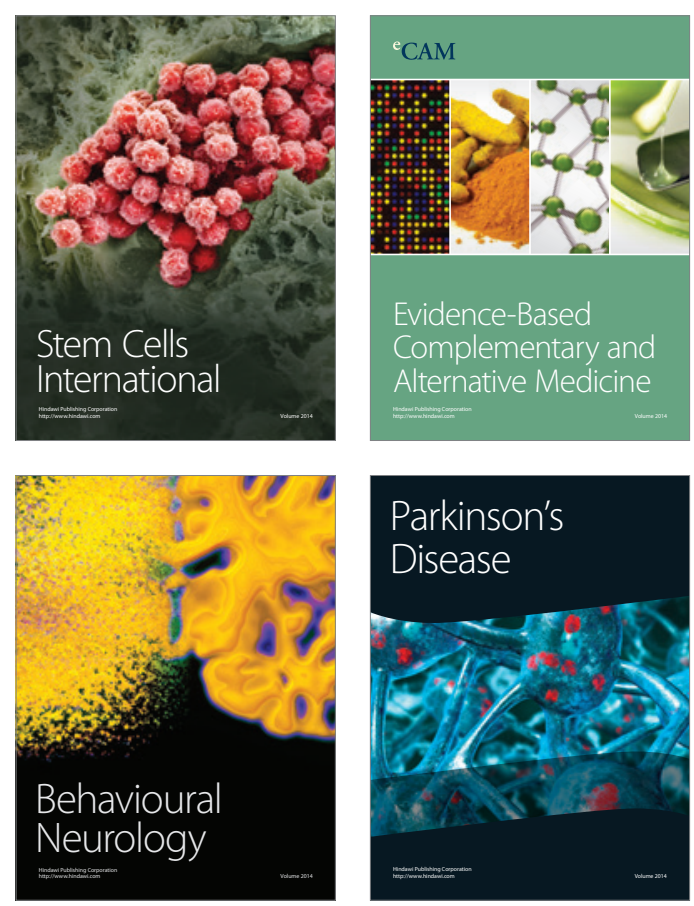

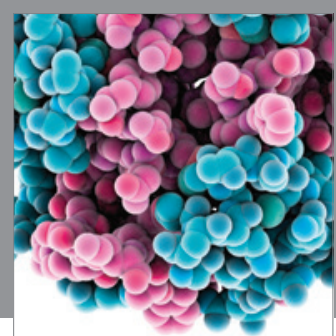

Journal of
Diabetes Research

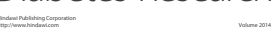

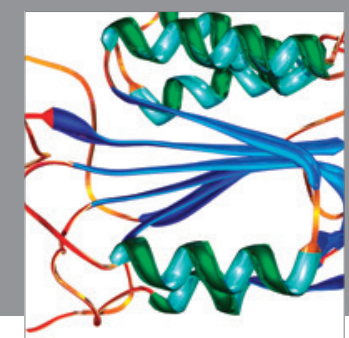

Disease Markers
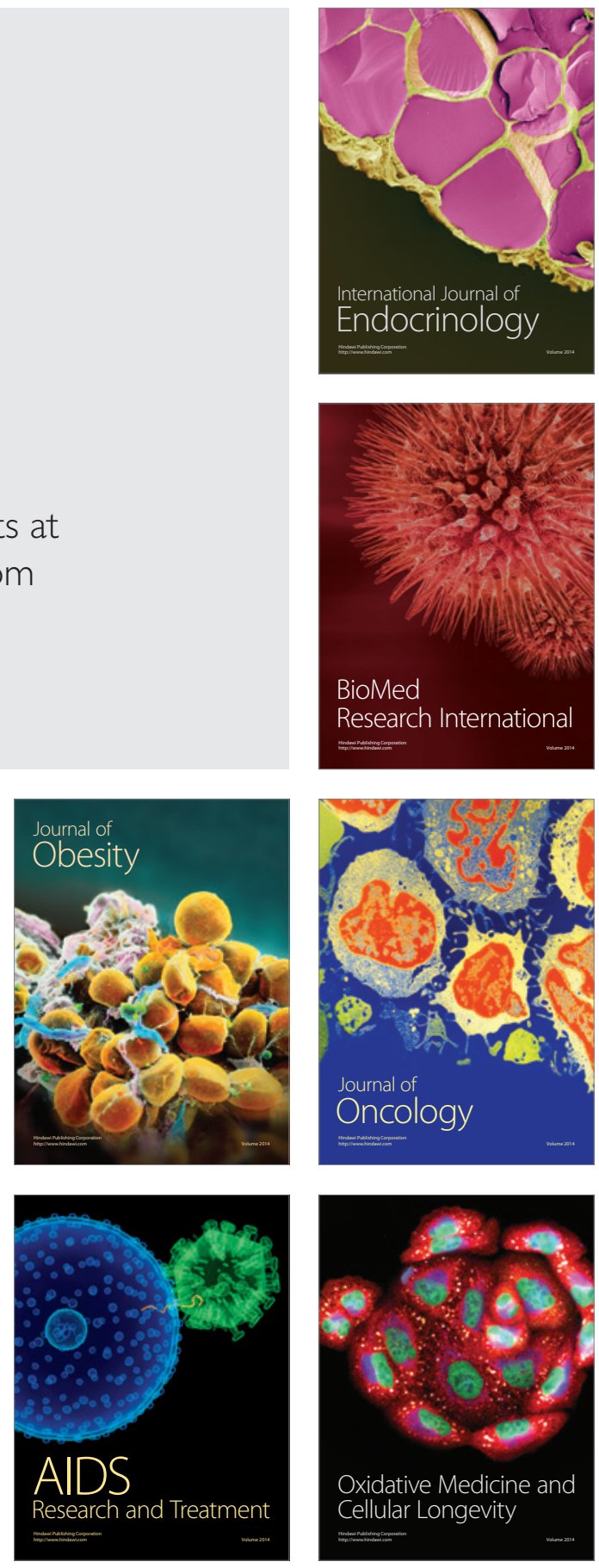